\section{In situ histochemical localisation of alkaloids and acetogenins in the endosperm and embryonic axis of Annona macroprophyllata Donn. Sm. seeds during germination}

\author{
A.E. Brechú-Franco, ${ }^{1}$ \\ G. Laguna-Hernández, ${ }^{1}$ \\ I. De la Cruz-Chacón, ${ }^{2}$ \\ A.R. González-Esquinca ${ }^{2}$
}

'Faculty of Sciences, National

Autonomous University of Mexico,

Coyoacán

${ }^{2}$ Institute of Biological Sciences, University of Sciences and Arts of Chiapas, Tuxtla Gtz., Chiapas, Mexico

\begin{abstract}
Currently, the Annonaceae family is characterised by the production of acetogenins (ACGs), and also by the biosynthesis of alkaloids, primarily benzylisoquinolines derived from tyrosine. The objective of this study was to confirm the presence of alkaloids and acetogenins in the idioblasts of the endosperm and the embryonic axis of $A$. macroprophyllata seeds in germination. The Dragendorff, Dittmar, Ellram, and Lugol reagents were used to test for alkaloids, and Kedde's reagent was used to determine the presence of acetogenins in fresh sections of the endosperm and embryonic axis of seeds after twelve days of germination. A positive reaction was observed for all the reagents, and the presence of alkaloids and acetogenins was confirmed in the idioblasts of the endosperm and those involved in the differentiation of the embryonic axis of the developing seedling. We concluded that the idioblasts store both metabolites, acetogenins and alkaloids. Beginning at differentiation, the idioblasts of the embryonic axis simultaneously biosynthesise acetogenins and alkaloids that are characteristic of the species during the development of the seedling. The method used here can be applied to histochemically confirm the presence of acetogenins and alkaloids in tissues and structures of the plant in different stages of its life cycle.
\end{abstract}

\section{Introduction}

Currently, the Annonaceae family is exclusively characterised by the production of compounds of a type of polyketide known as annonaceous acetogenins (ACGs), which have a wide range of biological activities. ${ }^{1-5}$ The majority of acetogenins are characterised by a long alkyl chain that typically contains tetrahydrofuran or tetrahydropyran rings, as well as a terminal lactone ring, generally in the form of an unsaturated $g$-lactone. This lactone produces a pink-coloured reaction in response to Kedde's reagent, which is used for staining in thin-layer chromatography procedures ${ }^{2,6-8}$ and this reaction is also observed in situ in fresh sections of $A$. macroprophyllata Donn. Sm. endosperm. ${ }^{9}$ The Annonaceae family also biosynthesise alkaloids, primarily benzylisoquinolines derived from tyrosine, of which more than 500 structures (mainly aporphines) have been reported in the family. These aporphinic alkaloids confer antimicrobial and insecticidal properties. ${ }^{10}$ Liriodenine is an alkaloid that shows a high recurrence in the Annonaceae family. ${ }^{5}$ Phytochemical studies of A. macroprophyllata have determined that the biosynthesis of liriodenine begins during seed germination and increases with the development of the seedling. Liriodenine also seems to have biological significance as a constitutive antimicrobial defence molecule. ${ }^{11}$ The acetogenins laherradurin, rolliniastatin-2 and cherimolin-2 have also been isolated from the seeds of this species. ${ }^{12-14}$ Recently, the in situ localisation of acetogenins in the idioblasts of the endosperm of the seeds of this species was reported. ${ }^{9}$ At the same time, the presence of molecules with a positive golden/browncoloured reaction in response to Lugol's reagent was observed in these idioblasts. Due to this result, the association between alkaloids and acetogenins was proposed. ${ }^{9}$. Furthermore, reports of the in situ localisation of alkaloids in other families have been published..$^{15-17}$ The objective of this work was to histochemically confirm the in situ localisation of acetogenins with Kedde's reagent and of alkaloids with the Lugol, Dragendorff, Dittmar, and Ellram reagents in the endosperm and the embryonic axis of $A$. macroprophyllata Donn. Sm. seeds during germination.

\section{Materials and Methods}

\section{Histochemistry}

The work was conducted with the same lot of $A$. macroprophyllata Donn Sm. seeds used in the studies of Laguna et al., ${ }^{9}$ and in the same reported conditions. The endosperm and embryonic axes after twelve days of germination were used to produce fresh, unfixed, 20 $\mu \mathrm{m}$-thick sections. The sections were cut between -14 and $-18^{\circ} \mathrm{C}$ using a Leica CM1510S cryostat. Consecutive sections of the endosperm and embryonic axis were mounted
Correspondence: Alicia Enriqueta BrechúFranco, Faculty of Sciences, National Autonomous University of Mexico, Av. Universidad 3000, Cd. Universitaria, Coyoacán, 04510 Ciudad de México, México D.F. Tel. +52.55 .56225431 - Fax: +52.55 .56224828 . E-mail: aliciae@ciencias.unam.mx

Key words: Annona seeds; acetogenins; Kedde's reagent; alkaloid's reagents; histochemistry; idioblasts; embryonic axis.

Contributions: all authors had substantial contributions to the acquisition, analysis, or interpretation of data for the work.

Conflict of interest: the authors declare no conflict of interest.

Acknowledgments: the authors thank Dr. Patricia Rivas Manzano and Biol. Carlos Tonatiuh Chavira Ramírez for technical assistance with the cryostat sectioning in the Laboratory of Tissue and Reproductive Biology, Faculty of Science, UNAM.

Received for publication: 23 September 2015. Accepted for publication: 19 December 2015.

This work is licensed under a Creative Commons Attribution-NonCommercial 4.0 International License (CC BY-NC 4.0).

(C) Copyright A.E. Brechú-Franco et al., 2016 Licensee PAGEPress, Italy

European Journal of Histochemistry 2016; 60:2568 doi:10.4081/ejh.2016.2568

on slides and rinsed with water three times for five minutes. The excess water was absorbed with filter paper to eliminate the adhesive gel, and several drops of the corresponding reagent were added to observe the reaction. Kedde's histochemical reaction was applied (10\% dinitrobenzoic acid and $2 \mathrm{~N} \mathrm{KOH}$ in $\mathrm{MeOH}$ ), which is specific for acetogenins and produces a positive pink/magenta-coloured reaction. ${ }^{9}$ The reactions used to detect alkaloids were conducted with the Dragendorff reagent $(8 \mathrm{~g}$ of bismuth subnitrate in $20 \mathrm{~mL}$ of nitric acid added to $22.7 \mathrm{~g}$ of potassium iodide dissolved in a small amount of water, followed by dilution of the mixture to $100 \mathrm{~mL}$ ), which produces a reddish-brown-coloured reaction; the Dittmar reagent $(1 \mathrm{~g}$ of potassium iodide, $1 \mathrm{~g}$ of sodium nitrite, $30 \mathrm{~mL}$ of hydrochloric acid, and $30 \mathrm{~mL}$ of distilled water), which produces a positive reddish-brown-coloured reaction; the Ellram reagent ( $1 \mathrm{~g}$ of vanillin in $100 \mathrm{~g}$ of $40 \%$ sulfuric acid), which produces a fuchsia or pink/magenta colour; ${ }^{15}$ and Lugol's iodine (0.5 g iodine and $2.0 \mathrm{~g}$ potassium iodide in $100 \mathrm{~mL}$ distilled water), which produces a blue/purple colour in response to starch ${ }^{18}$ and a goldenbrown colour in response to alkaloids. ${ }^{19}$ 


\section{Photomicrographs}

Photographs were obtained with a Canon Powershot A640 digital camera coupled to a Zeiss Axiostar microscope equipped with the Carl Zeiss Imaging System AxioVision program. The photomicrographic results were observed with A-Plan 10x/0.25 and A-Plan 40x/0.65 Ph2 objectives.

\section{Results}

In the endosperm of the seeds after twelve days of germination, ${ }^{9}$ the acetogenins present in the idioblasts of the periphery produced pink-magenta-coloured drops in the reaction with Kedde's reagent (Figure 1A). Lugol's reagent produced a golden-brown-coloured reaction in the same locations (Figure $1 \mathrm{~B}, \mathrm{C}$ ). This reaction was repeated with the Dragendorff reagent, which produced a golden-orange colour (Figure 1D), the Dittmar reagent, which produced a golden-brown colour (Figure 1E), and the Ellram reagent, which produced an olive colour (Figure 1F). In the embryonic axis, a positive, pink-magentacoloured reaction was observed in response to Kedde's reagent, which corresponded to the presence of acetogenins in the radicle and the hypocotyl. Scarce idioblasts were observed in the radicle, and an increased number were observed in the hypocotyl (Figure 2 A,B). In the embryonic axis sections, a positive reaction for alkaloids was produced in the idioblasts in response to Lugol's reagent (Figure 2C), as well as for the other reagents specific for alkaloids that contain iodine or iodide (such as the Dragendorff and Dittmar reagents). The stained areas for alkaloids were displaced in rows between the reserve parenchyma cells, which contained an abundance of amyloplasts. These amyloplasts can conceal idioblasts in some areas, because the amiloplasts acquire a dark-blue colour in response to the Lugol reagent and a dark-brown colour in response to the Dragendorff reagent (Figure 2D). A positive, reddish-brown-coloured reaction was observed in response to Dittmar's reagent in the idioblasts of the hypocotyl (Figure 2E). A reddish-pink colour localised to the idioblasts of the hypocotyl (Figure 2F) was observed in response to Ellram's reagent, which indicated a positive reaction for alkaloids.

\section{Discussion}

The positive reactions to Kedde's reagent and the alkaloid reagents were localised in the idioblasts of the endosperm, as well as in the hypocotyl and the radicle, which have conse- quently been identified as cells that simultaneously store acetogenins and alkaloids. The reaction of the Lugol reagent with the contents of the idioblasts in the endosperm reported by Laguna et al. ${ }^{9}$ was also shown in the test with Dragendorff's reagent, which has a composition similar to Lugol's. According to Yoder and Mahlberg, this finding confirms that the Lugol reagent may be used in the histochemical localisation of alkaloids. ${ }^{19}$ The positive reactions with the Dittmar and Ellram reagents confirm the alkaloid content of the idioblasts of the endosperm and the embryonic axis during the early developmental stages of this species. Specifically, the oxoaporphine alkaloid liriodenine was detected after twelve days of
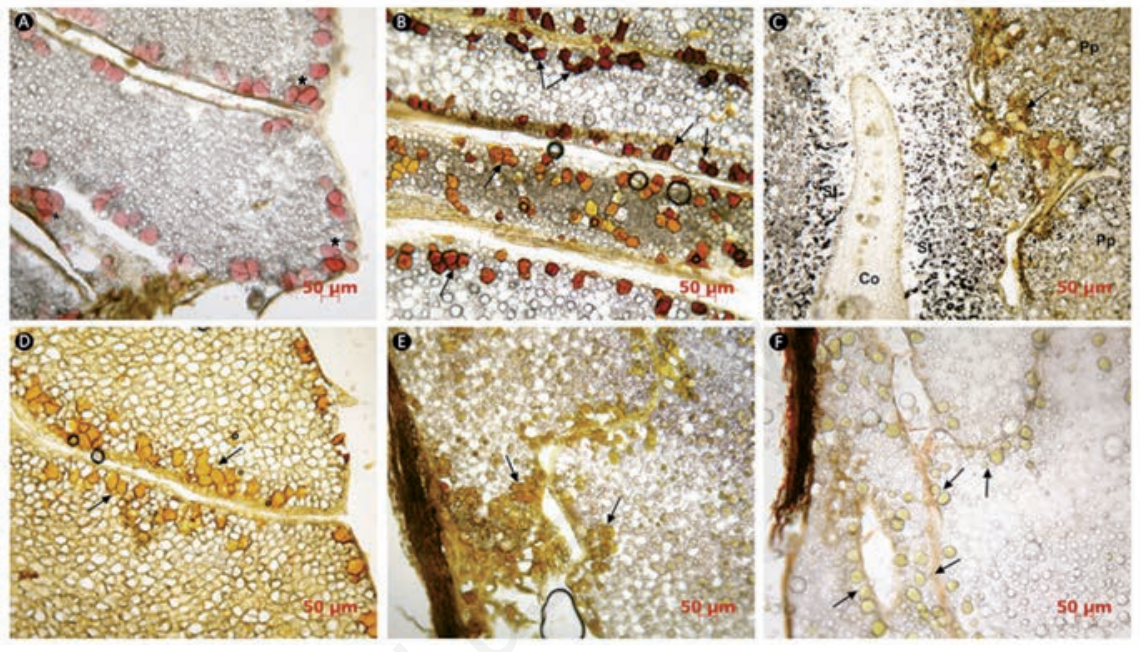

Figure 1. Endosperm sections after twelve days of germination. A) Kedde's reagent; pink/magenta-coloured drops indicate acetogenins (marked with the asterisks). B,C) Lugol's reagent; golden/brown-coloured drops indicate alkaloids, dark blue-coloured granules indicate starch. D) Dragendorff's reagent; gold/orange-coloured drops indicate alkaloids. E) Dittmar's reagent; golden/brown-coloured drops indicate alkaloids. F) Ellram's reagent; olive coloured drops indicate alkaloids. Arrows, alkaloid drops; St, starch granules; Pp, proteoplasts; Co, cotyledons.
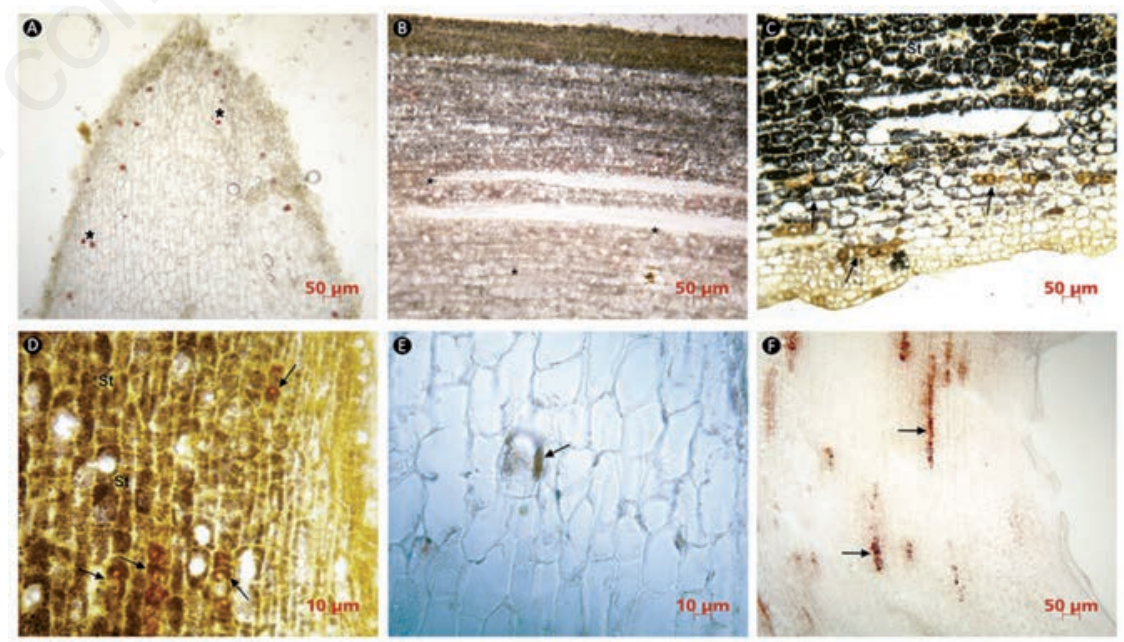

Figure 2. Embryonic axis sections after twelve days of germination. A,B) Radicle and hypocotyl exposed to Kedde's reagent; pink/magenta-coloured drops indicate acetogenins (marked with the asterisks). C) Hypocotyl exposed to Lugol's reagent; golden/browncoloured drops indicate alkaloids and dark blue-coloured granules indicate starch. D) Hypocotyl exposed to Dragendorff's reagent; golden/brown-coloured drops indicate alkaloids and dark brown-coloured granules indicate starch. E) Hypocotyl exposed to Dittmar's reagent; reddish-brown-coloured drops indicate alkaloids. F) Hypocotyl exposed to Ellram's reagent; reddish-pink-coloured drops indicate alkaloids. Arrows, alkaloid drops; St, starch granules. 
germination in the endosperm $\left(0.5 \mu \mathrm{g} \cdot \mathrm{g}^{-1}\right)$ and the embryonic axis $\left(1.13 \mu \mathrm{g} \cdot \mathrm{g}^{-1}\right)$. In the embryonic axis, the concentration of liriodenine increased in conjunction with development of the roots and stem (up to $700 \mu \mathrm{g} \cdot \mathrm{g}^{-1}$ ). ${ }^{20}$ In the present work, the localisation of unsaturated acetogenins in the embryonic axis in the developing idioblasts showed a similar concentration to that of the alkaloids, which is consistent with the presence of the pink-magenta reaction in response to Kedde's reagent and the golden-orange response corresponding to alkaloids with the Dittmar and Dragendorff reagents. The reddish-pink reaction colour produced by Ellram's reagent also confirms the presence of alkaloids in the idioblasts. The features of these reactions are consistent with the results reported by Fuhr and Mahlberg ${ }^{15}$ for the reference standards and for the alkaloids present in the glandular trichomes of leaves and the seedling epicotyl of Cannabis sativa. The reaction with Kedde's reagent demonstrated the presence of unsaturated acetogenins along the embryonic axis, radicle, and hypocotyl via a characteristic pink-magentacoloured reaction. In the same location in consecutive sections, however, the material also reacted with the alkaloid reagents (Dragendorff, Dittmar and Ellram). In this respect, the alkaloid reaction may correspond, in part, to liriodenine, which was detected for the first time in the A. macroprophyllata endosperm approximately five days after imbibition began. ${ }^{20}$ The early biosynthesis of liriodenine suggests a direct relationship with the degradation of reserve proteins, which, according to Müntz et al., ${ }^{21}$ are produced and stored during the intermediate and final stages of seed maturation and are converted into nitrogen deposits to be used later by the seedling during germination. Using Dragendorff's and Lugol's reagents in seeds after twelve days of germination, amyloplasts were localised in endosperm cells (adjacent to cotyledons) with signs of degradation, broken cell walls, no cytoplasmic content, and, in other cells, agglutinated amyloplasts (Figure 1C). In endosperm cells distal to the embryo, starch was not found in the plastids, and no signs of cellular degradation were present (Figure 1C). It is important to conduct a biochemical and ultrastructural study of the endosperm of $A$. macroprophyllata seeds during germination to determine the destination of the proteoplasts and their reserves because limited information exists regarding the genesis and interrelation of this type of plastid ${ }^{22}$ and its specific role in seedling development. Reports of the in situ localisation of alkaloids with Dragendorff's and Lugol's reagents in Catharanthus roseus ${ }^{19}$ and Cannabis sativa have been published. Corsi and Biasci ${ }^{16}$ have also demonstrated the presence of alkaloids in the secretory structures of
Conium maculatum via the Lugol and Wagner reactions, but they encountered certain difficulties due to the simultaneous staining of starch. In the present study, we observed that the Lugol reaction was produced not only in the idioblasts of the endosperm but also in differentiated idioblasts in the embryonic axis. However, in these embryonic-axis idioblasts, the reaction produced a lower concentration of the product, which coincides with a report that liriodenine biosynthesis begins during the first stages of development in the radicles and endosperm of the germinating seeds. ${ }^{20}$ The presence of amyloplasts in the parenchyma of the hypocotyl, which are stained dark blue, partially masked the idioblasts when they remained below the parenchyma cells, as Corsi and Biasci reported in their study. ${ }^{16}$ The correspondence of the alkaloid reactions observed in this study to the presence of these compounds was confirmed due to the results attained with Ellram's reagent. When Ellram's reagent was used, which is also used for alkaloid identification ${ }^{15,19}$ and does not contain potassium iodine or iodide, a positive olive and golden-brown-coloured reaction was observed in the idioblasts of the endosperm, and a specific reddish-pink-coloured reaction was observed in the idioblasts of the embryonic axis of the developing seedling. Thus, the compounds stored in idioblasts, together with acetogenins, were confirmed to be alkaloids by all tests using reagents specific for alkaloids.

A. macroprophyllata seeds contain idioblasts with a specific location in the endosperm and the embryonic axis of the developing seedling. The in situ localisation of alkaloids in the idioblasts with Lugol's, Dragendorff's, Dittmar's, and Ellram's reagents and the localisation of acetogenins with Kedde's reagent confirm that idioblasts store both types of metabolites and that the biosynthesis of these metabolites in the embryonic axis is simultaneous. The establishment of the idioblasts in the seedling begins with cellular differentiation and tissue organisation. Using this method, it is possible to histochemically detect acetogenins and/or alkaloids in the tissues or structures of Annona species in different stages of their life cycles.

\section{References}

1. Rupprecht JK, Hui YH, McLaughlin JL. Annonaceous acetogenins: a review. J Nat Prod 1990;53:237-78.

2. Cavé A, Cortes D, Figadère B, Laurens A, Pettit GR. Acetogenins from Annonaceae. In: W. Herz, G.W. Kirby, R.E.W. Moore, W. Steglich, C.H. Tamm (eds.) Progress in the chemistry of organic natural products.
Springer, 1997: pp. 81-288.

3. Zafra-Polo MC, Figadère B, Gallardo T, Tormo RJ, Cortés D. Natural acetogenins from Annonaceae, sinthesis and mechanism of action. Phytochemistry 1998;48:1087-117.

4. Alali FQ, Liu XX, McLaughlin JL. Annonaceus acetogenins: recent progress. J Nat Prod 1999;62:504-40.

5. Jumana S, Hasan CM, Rashid MA. Alakaloids from the stem bark of Miliusa velutina. Biochem Syst Ecol 2000;28:483-5.

6. Downum KR, Romeo JT, Stafford H. Phytochemical potential of tropical plants. New York, Springer: 1993.

7. Cunha MM, Nascimento FC, SantosPimenta LP, Boaventura MAD, Salas CE, Lopes MTP. Screening of cytitoxic activity in hexanic and ethanolic extacts of Rollinia laurifolia. Lat Am J Pharm 2009; 28:234-40.

8. Parellada EA, Ferrero M, Cartagena E, Bardón A, Neske A. Laherradurin, a natural stressor, stimulates QS mechanism involved in biofilm formation of a PAHs degrading bacterium. Int Biodeter Biodegrad 2013;85:78-84.

9. Laguna Hernández G, Brechú-Franco AE, De la Cruz-Chacón I, González-Esquinca AR. The histochemical detection of acetogenins and storage molecules in the endosperm of Annona macroprophyllata Donn Sm. seeds. Eur J Histochem 2015; 59:2502.

10. González-Esquinca AR, De la Cruz-Chacón I, Castro-Moreno M, Orozco-Castillo JA, Riley-Saldaña ChA. Alkaloids and acetogenins in Annonaceae development: biological considerations. Rev. Bras. Fruticult 2014;36:1-16.

11. De la Cruz-Chacón I, González-Esquinca AR, Guevara Fefer P, Jiménez García LF. Liriodenine, early antimicrobial defense in Annona diversifolia. Zeitt Naturforsch C 2011;66:377-384.

12. De la Cruz Chacón I. Acetogeninas bioactivas de Annona diversifolia Safford. PHd Thesis, Instituto Tecnológico de Tuxtla Gutiérrez, Chiapas: 2001.

13. Schlie-Guzmán MA, García Carranca A, González-Esquinca AR. In vitro and in vivo antiproliferative activity of laherradurin and cherimolin-2 of Annona diversifolia Saff. Phytother Res 2009;23:1128-33.

14. Luna Cazáres L, González-Esquinca AR. 2010 Susceptibility of bacteria and complete spheroplasts of Escherichia coli, Pseudomonas aeruginosa and Salmonella Typhi to rolliniastatin-2. Nat Prod Res 2010;24:1139-45.

15. Furr M, Mahlberg PG. Histochemical analyses of laticifers and glandular trichomes in Cannabis sativa. J Nat Prod 


\section{1;44:153-9.}

16. Corsi G, Biasci D. Secretory structures and localization of alkaloids in Conium maculatum L. (Apiaceae). Ann Bot 1998;81:157-62.

17. Jing H, Liu J, Liu H, Xin H. Histochemical investigation and kinds of alkaloids in leaves of different developmental stages in Thymus quinquecostatus. TheScientific WorldJo 2014;2014:839548.

18. Johansen DA. Plant microtechnique. New
York, McGraw-Hill: 1940.

19. Yoder LR, Mahlberg PG, Reactions of alkaloid and histochemical indicators in laticifers and specialized parenchyma cells of Catharanthus roseus (Apocynaceae). Am J Bot 1976;63:1167-73.

20. De la Cruz-Chacón I. González-Esquinca AR. Liriodenine alkaloid in Annona diversifolia during early development. Nat Prod Res 2012;26:42-9.
21. Müntz K, Belozersky MA, Dunaevsky YE, Schlereth A, Tiedemann J. Stored proteinases and the initiation of storage protein mobilisation in seeds during germination and seedling growth. J Exp Bot 2001;52:1741-52.

22. Newcomb E. Fine structure of protein-storing plastids in bean root tips. J Cell Biol 1967;33:143-63. 